\title{
Limitations in assessing the severity of aortic stenosis by Doppler gradients
}

\author{
RAGNAR DANIELSEN, ^ JAN ERIK NORDREHAUG, * LODVE STANGELAND, $\dagger$ \\ HARALD VIK-MO*
}

From the ${ }^{\star}$ Departments of Clinical Physiology and $†$ Cardiovascular Surgery, Haukeland Hospital, University of Bergen, Norway

SUMMARY Continuous wave Doppler echocardiography was performed before cardiac catheterisation in 69 consecutive patients with suspected aortic stenosis. Agreement between the maximum and the mean Doppler gradients and catheterisation gradients was good. Doppler echocardiography, however, systematically underestimated the maximum and mean gradients, particularly in the high range. Stepwise regression analysis of the small pressure difference between the two methods showed that it could not be explained by age, sex, stroke volume, differences in heart rate, ejection fraction, the presence of coronary artery disease, or severity of aortic regurgitation. There was a negative curvilinear correlation between the maximum and mean Doppler gradients and the aortic valve areas that were measured at catheterisation in patients with pure aortic stenosis. The degree of correlation decreased when patients with concomitant aortic regurgitation were included. The scatter of gradients above and below the correlation line was large and this was caused by low and high transvalvar flow.

These results show that the usefulness of Doppler gradients for judging the severity of aortic stenosis, both in relation to immediate diagnosis and follow up, is severely limited if transvalvar flow is not taken into account.

Several recent studies have shown the usefulness of Doppler echocardiography in assessing the systolic transvalvar pressure gradient in aortic stenosis. ${ }^{1-3}$ In these studies and in others ${ }^{4-6}$ variables were studied that might cause differences between calculated Doppler gradients and those measured at cardiac catheterisation, such as the age of the patient, the presence of aortic regurgitation, atrial fibrillation, low cardiac output, left ventricular function, associated coronary artery disease, and the experience of the operator with the Doppler technique. But such variables were only evaluated by subgroup analysis.

The decision on whether a patient should undergo aortic valve replacement is primarily based on the assessment of the aortic valve area, which is regarded as the most exact laboratory value. ${ }^{7}$ Although the systolic pressure gradient is an important determin-

Requests for reprints to Dr Ragnar Danielsen, Department of Clinical Physiology, N-5021 Haukeland Hospital, University of Bergen, Bergen, Norway.

Accepted for publication 16 December 1987 ant of the area, as reflected in the Gorlin formula, ${ }^{8}$ the calculated area is also influenced by the transvalvar flow.

The object of our study was to evaluate the usefulness of transvalvar gradients measured by Doppler echocardiography in predicting the severity of aortic stenosis (determined as the aortic valve area at catheterisation) in consecutive patients. To overcome some of the previous limitations of subgroup analysis we used stepwise regression analysis to assess the influence of physiological variables on the differences between the Doppler and catheterisation gradients.

\section{Patients and methods}

PATIENTS

Sixty nine consecutive patients (42 men) referred for cardiac catheterisation with suspected aortic stenosis, alone or in combination with other valve lesions, were evaluated from January 1985 to May 1986 . The mean (SD) age was $62(10)$ years (range 29-76 years); only eight patients were less than 50 years old. Fifteen patients also had aortic regurgitation (grades 
$3+$ or more) and two of these had mitral regurgitation (grades $3+$ or more). ${ }^{9}$ In addition, one patient had combined mitral stenosis and regurgitation, one had mitral regurgitation, and one had a prosthetic mitral valve.

Coronary artery stenoses ( $>50 \%$ luminal reduction) were found in $29(42 \%)$ patients; two of these were less than 60 . The angiographic ejection fraction ranged from 23 to $90 \%$ (mean (SD) $69(13) \%$ ) and the stroke volume from 30 to 179 (mean $75(27)$ ) $\mathrm{ml}$. The patients were in sinus rhythm, except for three with atrial fibrillation. To analyse the Doppler and catheterisation data we used an average of at least three heart cycles (10 cycles for the patients with atrial fibrillation). The Doppler examination was performed in all patients $<48$ hours before catheterisation (usually the day before). All patients were clinically stable in the time between the two examinations. The mean (SD) heart rates during the Doppler and catheterisation examinations were 73 (16) (range 49-112) and 73 (15) (range 45-112) beats/min. No patient was excluded from the study because of unsatisfactory Doppler data.

\section{DOPPLER ECHOCARDIOGRAPHY}

The continuous wave Doppler examination was performed with an IREX Meridian TM system with a $2 \mathrm{MHz}$ independent transducer and a combined 2-3/3.5 MHz Doppler and cross sectional echo transducer. Measurements of the ascending aortic jet were routinely done from the suprasternal or right parasternal windows with the independent transducer and from the apical window with the combined and/or independent transducer. Optimum Doppler recordings were obtained by locating the signal of highest audible frequency that gave the most clearly defined spectral velocity envelope and maximal velocity. Estimator functions were not used. We did not correct for a presumed angle between the Doppler beam and the maximum aortic jet.

The maximum velocity was assessed manually and converted to the instantaneous maximum pressure gradient by the modified Bernoulli equation ${ }^{10}: \triangle P=$ $4 \mathrm{~V}^{2}$, where $\triangle \mathbf{P}=$ pressure gradient $(\mathrm{mm} \mathrm{Hg})$ and $V$ $=$ velocity of the blood $(\mathrm{m} / \mathrm{s})$. The mean gradient was derived from the IREX Meridian measurement and an analysis software package that integrates the spectral velocity envelope at every $10 \mathrm{~ms}$ and calculates the mean of the instantaneous gradients derived from the above equation.

\section{CARDIAC CATHETERISATION}

The procedures were done from the percutaneous femoral approach. The left ventricle was catheterised by the retrograde or transseptal technique. Cardiac output was measured by the thermodilution method, left ventricular angiography was performed in the $30^{\circ}$ right oblique projection, and aortography in the $45^{\circ}$ left oblique position. Selective coronary angiography (Judkins technique) was done in all patients aged over 30 .

Intravascular pressure was recorded through fluid filled catheters with the transducer at the midaxillary level. In 55 patients the aortic valve gradient was measured from the immediate pullback pressure in the ascending aorta superimposed on the ventricular recording. Simultaneous pressure tracings obtained by the transseptal technique were used in 14 patients. We measured the instantaneous maximum, the traditional peak to peak, and the mean systolic pressure gradients. The aortic valve area was calculated from the Gorlin equation. ${ }^{8}$ In patients with clinically significant valve regurgitation we used single plane angiographic assessments of stroke volume $^{11}$ instead of the thermodilution data to calculate the area.

\section{STATISTICAL ANALYSIS}

Data are expressed as mean (1 SD). All tests were two tailed and the level of significance was set at $p<0.05$. Student's $t$ test was used to compare mean differences between groups. The agreement between Doppler and catheterisation gradients was evaluated according to the method of Altman and Bland. ${ }^{12} \mathrm{We}$ used stepwise regression analysis, forwards and backwards $^{13}$ (MINITAB statistical software ${ }^{14}$ ), to assess the possible influence of independent variables on the differences between Doppler and catheterisation gradients. A non-linear relation was derived for the association between pressure gradients and the aortic valve areas after logarithmic transformation to fit linear regression. ${ }^{14}$

\section{Results}

\section{AGREEMENT BETWEEN PRESSURE GRADIENTS}

There was good agreement between the maximum Doppler gradients and those measured by catheterisation (fig 1a). The maximum Doppler gradients ranged from 16 to $144 \mathrm{~mm} \mathrm{Hg}$ (mean 80 (33) $\mathrm{mmHg}$ ) and those at catheterisation from 19 to $182 \mathrm{~mm} \mathrm{Hg}$ (mean 85 (37) $\mathrm{mm} \mathrm{Hg}$ ). The mean Doppler gradients ranged from 10 to $102 \mathrm{~mm} \mathrm{Hg}$ (mean 51 (23) $\mathrm{mm} \mathrm{Hg}$ ) and the mean catheterisation gradients from 11 to 141 $\mathrm{mm} \mathrm{Hg}$ (mean $58(28) \mathrm{mm} \mathrm{Hg}$ ). Again these were in close agreement (fig 1b). For one obvious outlier, however, a mean catheterisation gradient of $141 \mathrm{~mm}$ $\mathrm{Hg}$ was estimated by Doppler to be $85 \mathrm{~mm} \mathrm{Hg}$. The corresponding maximum gradients were 182 and 148 $\mathrm{mm} \mathrm{Hg}$. The generally good agreement between the maximum and mean gradients obtained by the two 
methods also applied to the 15 patients with aortic stenosis and regurgitation (fig $1 a$ and $b$ ).

\section{DIFFERENCE BETWEEN PRESSURE GRADIENTS}

The maximal Doppler $(p<0.001)$ and catheterisation gradients $(p<0.001)$ were significantly higher and different from the peak to peak gradients at catheterisation, which ranged from 4 to $153 \mathrm{~mm} \mathrm{Hg}$ (mean 66 (35) $\mathrm{mm} \mathrm{Hg}$ ). Compared with the catheterisation gradients, the Doppler technique systematically underestimated both the maximum and the mean gradients, particularly in the high range. This caused on average a small but significant pressure difference between the two methods for
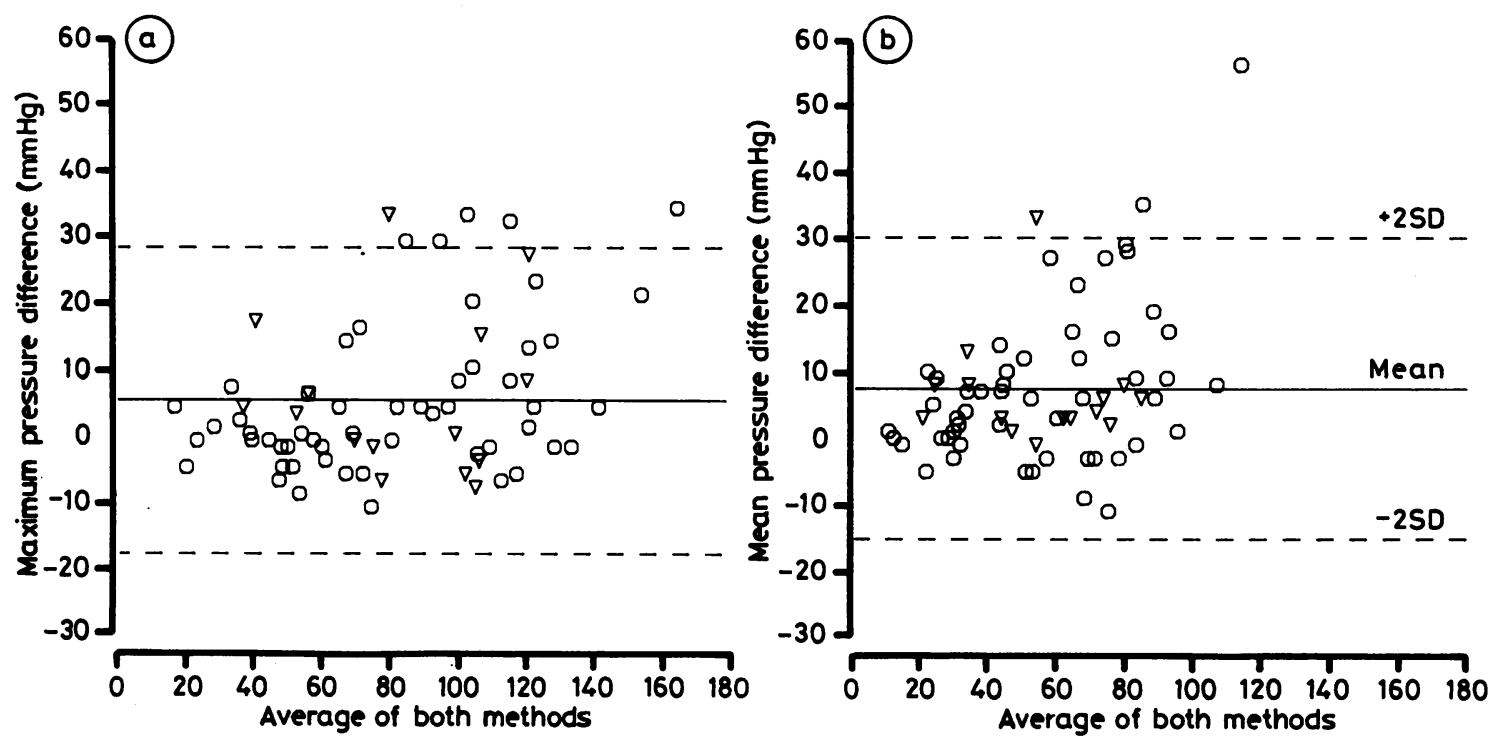

Fig 1 The difference between ( $a$ ) maximum and (b) mean catheterisation and Doppler systolic pressure gradients in relation to the average pressure measured by both methods. $\bigcirc$ pure aortic stenosis $(n=54) ; \Delta$ aortic stenosis and regurgitation (n $=$ 15).
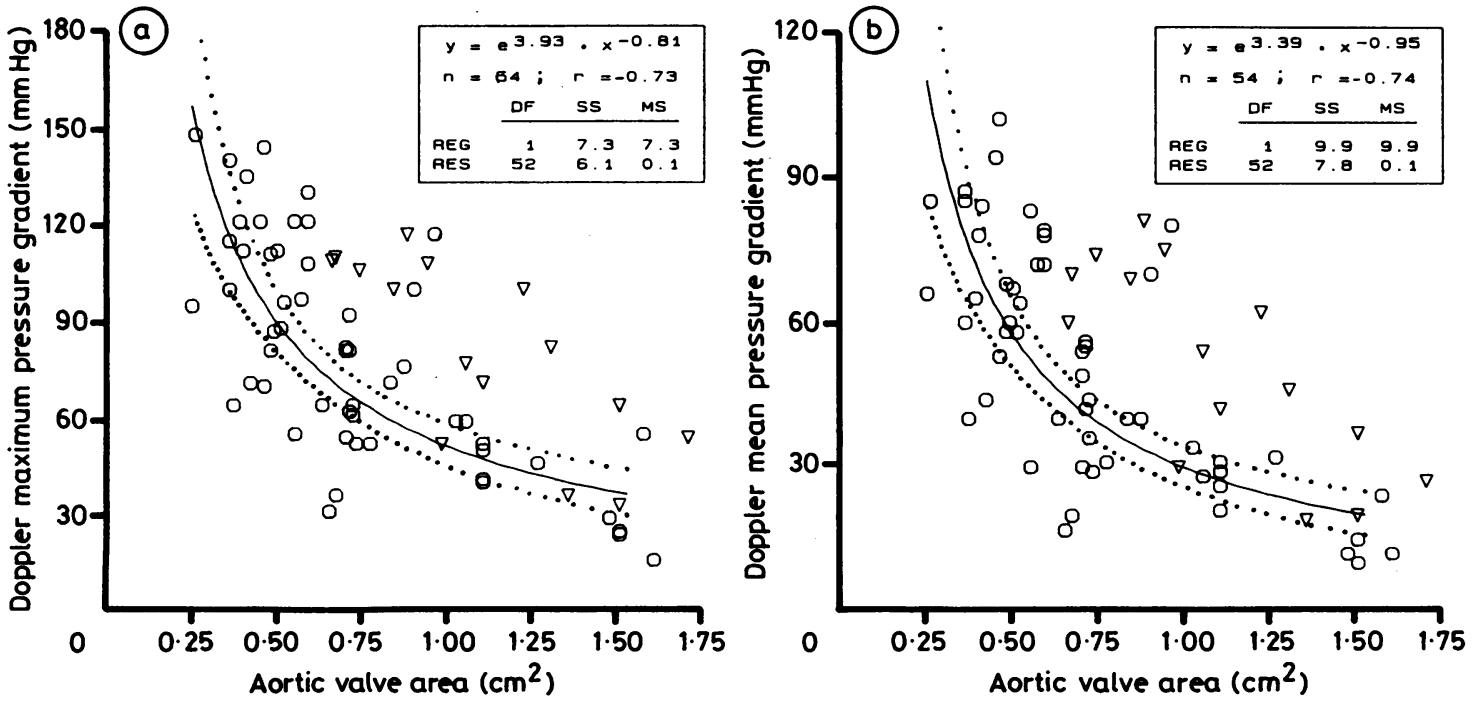

Fig 2 The relation of systolic pressure gradients to the aortic valve area. (a) Maximal Doppler gradients versus aortic valve areas. (b) Mean Doppler gradients versus aortic valve areas. The non-linear correlation with $95 \%$ confidence interval was calculated for patients with pure aortic stenosis only $(n=54) . D F$, degrees of freedom; MS, mean square; $S S$, sum of squares; REG, regression; RES, residual. See fig 1 for symbols. 
both the maximum $(p<0.001)$ and mean gradients $(\mathrm{p}<0.001)$. In stepwise regression analysis this difference could not be explained by age, sex, stroke volume, ejection fraction, the presence of coronary artery disease, severity of aortic regurgitation, or differences in heart rate between the two examinations. There were too few (three) patients with atrial fibrillation for this feature to be included as an independent variable in the stepwise regression analysis.

\section{RELATION OF PRESSURE GRADIENTS TO AORTIC} VALVE AREAS

As predicted by the Gorlin equation, ${ }^{8}$ a negative curvilinear function described the relation between the maximum $(r=-0.73)$ and the mean $(r=-0.74)$ Doppler gradients and the aortic valve areas at catheterisation in the patients with pure aortic stenosis ( $n=54)$ (fig $2 a$ and $b$ ). This correlation decreased when the patients who also had aortic regurgitation $(n=15)$ were included $(r=-0.65$ and -0.66 respectively). There was a large scatter of pressure gradients above and below the correlation lines. For critical areas $\leqslant 0.75 \mathrm{~cm}^{2}$ the range of maximum and mean Doppler gradients was 31 to 144 and 17 to $102 \mathrm{~mm} \mathrm{Hg}$ respectively. The scatter of the maximal and peak to peak catheterisation gradients in relation to the areas was similar (detailed data not shown).

\section{Discussion}

The area of the aortic valve is usually considered to be more reliable than the systolic pressure gradient in predicting the need for valve replacement in patients with aortic stenosis. ${ }^{7}$ Although Doppler echocardiography accurately detected the transvalvar gradients, the curvilinear relation between the pressure gradients and the aortic valve areas was less exact. In fact, the range of Doppler gradients for a given area was wide. According to the Gorlin formula, ${ }^{8}$ this scatter of gradients is the result of variations in transvalvar flow. It could not be explained by the small differences between the Doppler and catheterisation gradients. When transvalvar flow is low small valve areas can be seen with low gradients, especially in patients with poorly contracting or small hypertrophied left ventricles. Valve areas that are larger than predicted from pressure gradients are seen when the transvalvar flow is increased, as in patients with aortic regurgitation and other high output cardiac states. ${ }^{710}$

Although the presence of aortic regurgitation, or other causes of a high stroke volume, did not significantly influence the measurement of Doppler pressure gradients in this study, the aortic valve areas could not be predicted from the pressure gradients alone in such patients. Thus high or low transvalvar flow severely limits the accuracy of the Doppler method in assessing the aortic stenosis. Furthermore, during follow up an unchanged gradient does not exclude the possibility of a significant decrease of the aortic valve area. However, the development of Doppler techniques that non-invasively assess the cardiac output ${ }^{15}$ or the actual aortic valve area ${ }^{17-19}$ largely overcomes these limitations of the Doppler method.

Despite the close agreement between the transvalvar pressure gradients obtained by Doppler and catheterisation the Doppler technique systematically underestimated the results of catheterisation data, particularly gradients in the high range. This caused the Doppler gradients to be on the average slightly, but significantly, lower than those at catheterisation. In stepwise regression analysis the small pressure difference between the two methods was unrelated to age, sex, stroke volume, ejection fraction, the presence of aortic regurgitation, coronary artery disease, or differences in heart rate between the two examinations.

Previous studies that assessed various subgroups alone, ${ }^{2}$ or combined with linear regression analysis ${ }^{45}$ did not find that the correlation coefficients were influenced by low cardiac output, left ventricular function, or associated coronary artery disease. A recent study, however, that used the same statistical method, found that experience with the Doppler technique, the presence of aortic regurgitation, and atrial fibrillation did. ${ }^{6}$ Stepwise regression analysis avoids splitting the study population into small subgroups and thus may partly explain why our results were different. The good agreement between the Doppler and catheterisation gradients in this study might, however, be partly the result of having few patients in the study group with atrial fibrillation. On the other hand, we did include consecutive patients and did not exclude any Doppler data from the analysis. Thus we found that experience with the technique and the use of several transducer positions seem to be more important than other physiological factors for obtaining a reliable Doppler gradient.

Technical errors may contribute to differences in measuring gradients. These include the fidelity of the fluid filled catheter system, catheter induced artefacts, and phase shifts between non-simultaneous measurements of left ventricular and aortic pressure. ${ }^{4}$ On the other hand, the Doppler results are influenced by angle errors, experience, the use of multiple transducer positions, and assumptions in the modified Bernoulli equation. ${ }^{210}$ Although the Doppler and catheterisation measurements were not simultaneous in our study, this seems to have had 
little effect on their compatibility.

We conclude that Doppler echocardiography is accurate in assessing the systolic transvalvar pressure gradients in patients with aortic stenosis and is less influenced by physiological factors than previously reported. Although there is a significant negative curvilinear relation between Doppler gradients and aortic valve area, we found that, in contrast with earlier reports, ${ }^{35}$ this association is not accurate enough to be the basis for a decision about valve replacment if the transvalvar flow is not also assessed.

\section{References}

1 Agatson AS, Chengot M, Rao A, Hildner F, Samet P. Doppler diagnosis of valvular aortic stenosis in patients over 60 years of age. Am J Cardiol 1985; 56:106-9.

2 Hegrenœs L, Hatle L. Aortic stenosis in adults. Noninvasive estimation of pressure differences by continuous wave Doppler echocardiography. Br Heart J 1985;54:396-404.

3 Simpson IA, Houston AB, Sheldon CD, Hutton I, Lawrie TDV. Clinical value of Doppler echocardiography in the assessment of adults with aortic stenosis. Br Heart $J$ 1985;53:636-9.

4 Currie PJ, Seward JB, Reeder GS, et al. Continuouswave Doppler echocardiographic assessment of severity of calcific aortic stenosis: a simultaneous Doppler-catheter correlative study in 100 adult patients. Circulation 1985;71:1162-9.

5 Yeager M, Yock PG, Popp RL. Comparison of Doppler-derived pressure gradient to that determined at cardiac catheterization in adults with aortic valve stenosis: implications for management. Am J Cardiol 1986;57:644-8.

6 Panidis IP, Mintz GS, Ross J. Value and limitations of Doppler ultrasound in the evaluation of aortic stenosis: a statistical analysis of 70 consecutive patients. Am Heart $J$ 1986;112:150-8.

7 Braunwald E. Aortic stenosis. In: Braunwald E, ed. Heart disease: a textbook of cardiovascular medicine.
Philadelphia: WB Saunders, 1984:1095-105.

8 Gorlin R, Gorlin SG. Hydraulic formula for calculation of the area of the stenotic mitral valve, other cardiac valves, and central circulatory shunts. I. Am Heart J 1951;41:1-29.

9 Croft $\mathrm{CH}$, Libscomb K, Mathis $\mathrm{K}$, et al. Limitations of qualitative angiographic grading in aortic and mitral regurgitation. Am J Cardiol 1984;53:1593-8.

10 Hatle L, Angelsen B. Doppler ultrasound in cardiology. Philadelphia: Lea and Febiger, 1985:8-31 and 124-43.

11 Kennedy JW, Trenholm SE, Kasser IS. Left ventricular volume and mass from single-plane cineangiocardiograms. A comparison of anteroposterior and right anterior oblique methods. $\mathrm{Am}$ Heart J 1970;80:343-52.

12 Altman DG, Bland JM. Measurements in medicine: the analysis of method comparison studies. The Statistician 1983;32:307-17.

13 Draper NR, Smith H. Applied regression analysis. New York: John Wiley and Sons, 1966:163-77.

14 Ryan BF, Joiner BL, Ryan TA. MINIT AB handbook. Boston: Duxbury Press, 1985:218-59 and 358.

15 Lewis JF, Kuo LC, Nelson JG, Limacher MC, Quinones MA. Pulsed Doppler echocardiographic determination of stroke volume and cardiac output: clinical validation of two new methods using the apical window. Circulation 1984;70:425-31.

16 Zhang Y, Nitter-Hauge S, Ihlen H, Myhre E. Doppler echocardiographic measurement of cardiac output using the mitral orifice method. $\mathrm{Br}$ Heart $J$ 1985;53:130-6.

17 Skjœrpe T, Hegranœs L, Hatle L. Noninvasive estimation of valve area in patients with aortic stenosis by Doppler ultrasound and two-dimensional echocardiography. Circulation 1985;72:810-8.

18 Zhang $Y$, Myhre E, Nitter-Hauge S. Noninvasive quantification of the aortic valve area in aortic stenosis by Doppler echocardiography. Eur Heart J 1985; 6:992-8.

19 Richards KL, Cannon SR, Miller JF, Crawford MH. Calculation of aortic valve area by Doppler echocardiography: a direct application of the continuity equation. Circulation 1986;73:964-9. 